\title{
Planned future high-resolution radio observations of the GOODS-N field
}

\author{
Tom Muxlow ${ }^{1}$ \\ Jodrell Bank Centre For Astrophysics, University of Manchester, UK. \\ E-mail: twbmejb.man.ac.uk
}

\section{Ian Smail}

University of Durham, UK.

E-mail: ian.smailedurham.ac.uk

\section{lan McHardy}

University of Southampton, UK.

E-mail: i.m.mchardy@soton.ac.uk

\section{\& the e-MERGE Consortium}

In order to statistically characterize the nature of the sub- $\mu \mathrm{Jy}$ radio population - the target objects for the SKA, an ambitious Legacy survey has been proposed ( $e$-MERGE) to exploit $e$-MERLIN's unique combination of sensitivity and spatial resolution to study the formation and evolution of starforming galaxies and AGN out to redshifts of $z>5$. These observations will provide a powerful, obscuration-independent tool for measuring the massive star formation and AGN activity in highredshift galaxies, hence tracing the development of the stellar populations and the black hole growth in the first massive galaxies. To achieve these goals, we have developed a strategy comprising three nested tiers, which together provide a single, coherent survey addressing fundamental questions about the formation and joint evolution of AGN and galaxies.

One of the proposed tiers comprises very deep survey of some of the faintest radio starburst galaxies and AGN systems in the GOODS-N region and is designed to directly address the following key science drivers: (1) To extend the star-formation (SF) density history to redshifts $>5$ and thus trace the evolution of star-formation through cosmic time. (2) To determine the contribution of AGN to activity in the distant galaxy population and separate AGN from starbursts by high resolution multifrequency observations. (3) To determine the role of AGN in driving and controlling the SF processes

The 9th European VLBI Network Symposium on

The role of VLBI in the Golden Age for Radio Astronomy and EVN Users Meeting

Bologna, Italy

September 23-26, 2008

\footnotetext{
$1 \quad$ Speaker
} 


\section{Introduction}

One of the key goals of modern cosmology is to understand the formation and evolution of the whole galaxy population. There has been good progress in addressing this problem using optical and near-infrared surveys over the past two decades culminating in the first attempts to determine the integrated star formation history of the Universe [7], [9]. However, to reliably interpret this evolution we need to break down the integrated history of star formation, and independently track the star formation activity building the stellar mass in the rotationally- and pressure-supported structural components of galaxies (disks and spheroids or bulges), as well as the variation of these processes with redshift, galaxy mass and environment. This separation is essential if fundamental differences exist in the physical processes responsible for the star formation activity which builds disks and spheroids, due to differing timescales, metallicity or density dependence of the modes of star formation. Attempts to do this, using high-resolution ( 100-mas) optical imaging from Hubble Space Telescope (HST) have suggested that the strong increase seen in the integrated star formation rate in the Universe out to $\mathrm{z} \sim 1$ is driven by increasing activity in disk galaxies [8]. Nevertheless, the presence of significant numbers of blue spheroids suggests that some star formation is occurring in this component [10]. However, these studies suffer from a major weakness: that they miss the intense star formation activity in the most dust obscured environments within galaxies. There is thus an urgent need to apply a dust-insensitive tracer of star formation to provide a spatially resolved view of galaxy formation to identify and so determine which of the myriad of physical processes influencing the evolution of galaxies are critical.

Radio continuum observations, and the existence of the radio-far-infrared correlation, provide us with the tool we need to resolve and measure the massive star formation in high-redshift galaxies and so track the build up of their stellar populations. To spatially resolve the star formation activity in disks and spheroids we need centimetric radio observations with sub$\mathrm{kpc} / \mathrm{kpc}$ spatial resolution at $\mathrm{z}>1$, corresponding to 50-100 milliarcseconds.

\section{The Original Radio Study}

From observations made in the mid 1990s, deep MERLIN+VLA L-Band imaging was performed on a $10 \times 10$ arcminute field centered on the Hubble Deep Field North (HDFN). Within this field, 92 radio sources were detected by the VLA above a completeness limit of $40 \mu \mathrm{Jy}$. Deep, high-resolution MERLIN+VLA postage-stamp combination images were made of each detected source. This study is described in [11]; a brief summary of some of the conclusions are shown below:

- Combination L-Band MERLIN+VLA observations can morphologically distinguish AGN from starburst systems.

- Below $\sim 70 \mu \mathrm{Jy}$ the radio population becomes dominated by powerful star-forming galaxies typically at $z<1.5$ and with star-formations rates of many times those seen in nearby star-forming galaxies.

- Around $15 \%$ of the $\mu \mathrm{Jy}$ radio source population are found at redshifts higher that $\sim 1.5$, many of which are also identified as sub-mm sources. 
- Some of the most luminous starburst systems also show evidence for powerful embedded AGN.

A number of the brightest radio sources in the central part of the field were also imaged a very high angular resolution with the EVN [4].

\subsection{Recent Developments}

A new high-resolution, wide-field combination LBand image has been made covering a complete field 8.5 x 8.5 arcminutes centered on the HDFN. This field overlies part of the GOODS$\mathrm{N}$ field which has received extensive multi-wavelength coverage in recent years.

Utilizing ancillary data from GOODS-N, radio emission at the level of a few $\mu \mathrm{Jy}$ has been statistically detected associated with ACS galaxies brighter than a z-band magnitude of 26. Radio emission was searched for within a radius of 0.75 arcseconds of the

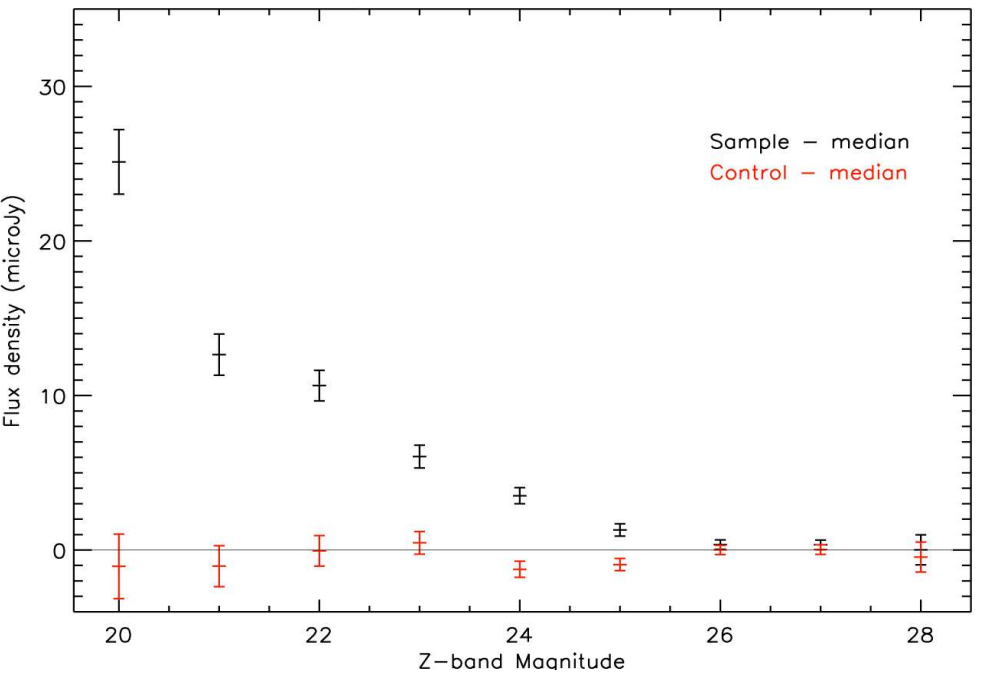
positions of 13030 z-band galaxies - excluding known brighter $\mu$ Jy sources identified in [11], and close galaxy pairs. This is shown in Figure 1.

The majority of galaxies statistically detected in the radio are identified as extended starburst systems with average properties similar to those star-forming galaxies studied individually at higher flux density levels. This study has allowed the investigation of the established infraredradio correlation to very low flux densities [1].

With regard to those objects within this field which have been identified as sub-mm sources, high-resolution radio imaging has been shown to be a powerful diagnostic tool and has been used to discriminate between $\mathrm{AGN}$ and star formation in the diverse sub-mm galaxy (SMG) populations at $\mathrm{z} 2$.

Two thirds of the SMGs show resolved radio morphologies extending to $\sim 1$ " which is interpreted as bolometric output arising in extended, faint, dust obscured regions that are forming stars at close to their Eddington limit. [3]. In addition, MERLIN +VLA imaging has identified some sub-mm faint radio galaxies within GOODS-N as part of the radio-AGN population which were previously misidentified as starbursts at other wavelengths [2]. 


\section{The Proposed Ultra-Deep Study of GOODS-N}

This is part of a tiered $e$-MERLIN Legacy proposal - the $e$-MERGE Survey which is designed to study the formation and evolution of star-forming galaxies and AGN out to redshifts $>5$.

- The e-MERlin Galaxy Evolution Survey

Tier 0 - Imaging radio emission from normal galaxies out to $\mathrm{z} \sim 5$

Deep imaging around clusters to utilise amplification by lensing

Tier 1 - A very deep directed survey of the $\mu \mathrm{Jy}$ radio source population

Deep imaging of the $\mu J y$ radio source population in GOODS-N

Tier $2-A$ reliable cosmic census of starburst and AGN populations

Medium depth imaging over a number of fields (total area $\sim 2$ sq. degrees)

The combination of these tiers will ensure a full sampling of the active (AGN) and star-forming galaxy radio luminosity function out to $\mathrm{z} \sim 5$. The $e$-MERGE consortium comprises more than 60 CO-I astronomers from 9 countries. Tier 0 is led by Ian Smail [Durham], Tier 1 by Tom Muxlow [Manchester], and Tier 2 by Ian McHardy [Southampton]. Around 2400 hours of $e$ MERLIN time have been proposed.

Tiers 0 and 1 have been approved in full and are now awaiting scheduling. Tier 2 was not approved, but will be the subject of a re-scoping exercise following which it will be proposed through the conventional PATT route.

Tier 1 represents a very deep survey of some of the faintest radio starburst galaxies and AGN systems in the GOODS-N region and is designed to directly address the following key science drivers:

- To extend the star-formation (SF) density history to redshifts $>5$ and thus trace the evolution of star-formation through cosmic time.

- To determine the contribution of AGN to activity in the distant galaxy population and separate AGN from starbursts by high resolution multi-frequency observations.

- To determine the role of AGN in driving and controlling the SF processes

- To statistically characterize the nature of the sub- $\mu$ Jy radio population - the target objects for the SKA.

\subsection{The New Tier-1 Radio Images}

$e$-MERLIN observations at both L-Band and C-Band are proposed for this tier.

\subsubsection{L-Band Imaging}

$e$-MERLIN will exceed the depth of the existing MERLIN+VLA combination map in just 24 hours of on-source integration. The new L-Band image will consist of a single pointing centre 
with 20 full tracks including Lovell telescope. For the central ultra-deep 10 arcminute field this will deliver a sensitivity of $1 \sigma \sim 500 \mathrm{nJy} / \mathrm{beam}$. In the surrounding outer 30 arcminute field a noise level of $1 \sigma \sim 1 \mu \mathrm{Jy} / \mathrm{beam}$ will be achieved. This should be compared with the original study of 18 full MERLIN tracks +42 hours of VLA A-array which imaged the central 10 arcminute field only to a depth of $1 \sigma \sim 3.3 \mu \mathrm{Jy} / \mathrm{beam}$, The original central field contained 92 sources $>40 \mu \mathrm{Jy}$ ( 60 starburst galaxies and $32 \mathrm{AGN}$ systems). By comparison, within the inner 10 arcminute field, $e$-MERLIN will image $\sim 850$ individual starburst and AGN with an angular resolution of $\sim 200$ milliarcseconds, complete to $\sim 3 \mu \mathrm{Jy}$ ( $>\mathbf{1 0}$ times deeper than the original 2005 study [11]). In the surrounding 800 square arcminutes, $e$-MERLIN will image $\sim 2500$ starforming galaxies and $\sim 1200$ AGN brighter than $\sim 6 \mu \mathrm{Jy}$. This represents a $>50 \mathrm{x}$ increase in source numbers over the original study.

Additional matching EVLA L-Band A-array data ( $40 \mathrm{hrs})$ will be sought in order to provide extra short-spacing coverage for the recovery of any very extended sources. This will also reduce the noise levels in the images by a factor of $\sim 1.5$ to $\sim 330 \& \sim 670 \mathrm{nJy} /$ beam in the inner 10 arcminute and outer 30 arcminute fields respectively.

\subsubsection{C-Band Imaging}

A new mosaiced C-Band image including the Lovell Telescope has also been proposed. This will consist of 7 pointing centres each with 3 long tracks in a mosaic pattern optimised for the Lovell 3 arcminute beam (Figure 2). This will produce a new ultra-deep C-Band image covering the central ultra-deep 10-arcminute field at L-Band. The C-Band field will consist of two closely matched regions:

- An inner 6' diameter field with a noise level $1 \sigma \sim 500 \mathrm{nJy} / \mathrm{beam}$

- A surrounding annulus between diameters of 6-10' with a noise level $1 \sigma$ 700nJy/beam

The complete ultra-deep C-Band image will have an angular resolution of 40 milliarcseconds. Additional C-Band EVLA data will be sought (6 hours per pointing) which increases the sensitivity by a factor of $\sim 1.5$ and adds short-spacing $u v$ -

coverage to recover the very heavily resolved radio structures with sizes $>1.2$ arcseconds.

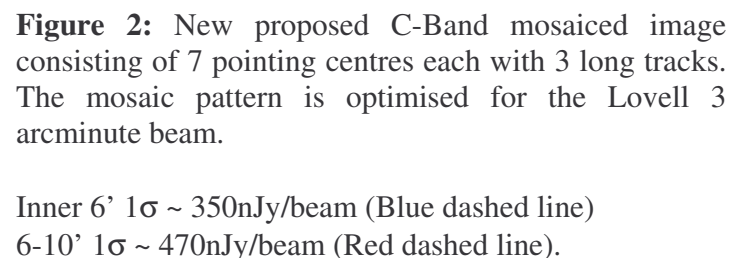

Figure 2: New proposed C-Band mosaiced image consisting of 7 pointing centres each with 3 long tracks. The mosaic pattern is optimised for the Lovell 3 arcminute beam.

Inner 6' $1 \sigma \sim 350$ nJy/beam (Blue dashed line)

6-10' $1 \sigma \sim 470 \mathrm{nJy} /$ beam (Red dashed line).

The ultra-deep C-Band image will:

- Map the star-forming regions in great detail

- Separate and disentangle the AGN and starburst components of emission

- Study the role that the AGN play in controlling star-formation via feedback - on subkpc scales for several hundred galaxies.

- Produce total intensity and spectral imaging across each detected system. 
More detailed jet-induced star-formation studies will also require the additional very high angular resolution of the EVN at L-Band in combination with $e$-MERLIN which will compliment these new C-Band images.

\section{Star-formation History from Starburst Luminosities}

For that population of very weak radio sources which we can at present only study statistically, $e$-MERLIN, EVLA and VLBI should image $\sim 1000$ systems to $\sim 3-4 \mu \mathrm{Jy}$ at L-band with $150-200$ at high redshift in a single central field. Additionally, many thousands of galaxies with radio flux densities $<1 \mu \mathrm{Jy}$ will be studied statistically.

Figure 3 shows the co-moving star-formation rate density (SFRD) of the Universe as a function of redshift from L-Band radio studies by Seymour [13], Haarsma et al [5], \& UV, H $\alpha$, Far-IR..., Hopkins [6]. The new L-Band results from the $e$ MERGE survey will provide data for several thousand more sources, tightening the Seymour error bars by a factor $\sim 4$.

Figure 3: Co-moving star-formation rate density as a function of redshift. L-Band radio studies from Seymour et al [13] [Red], Haarsma et al [5] [Blue], UV, H $\alpha$, Far-IR... from Hopkins [6] [Grey].

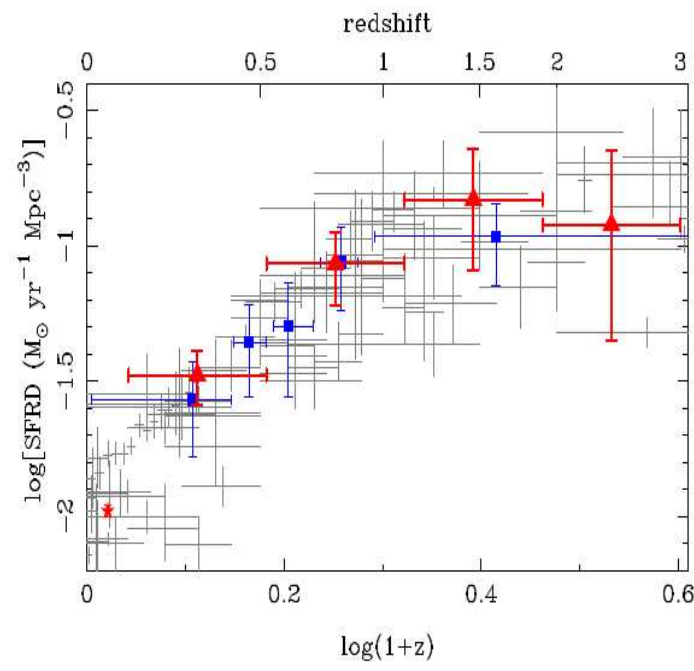

Sub-mm starburst systems at high redshift show evidence for substantial AGN activity. The EVN will play a pivotal role in probing the role of AGN/jets in such systems and in estimating the AGN contamination to the radio emission from the starburst.

\section{Summary}

If approved, observations will require full specifications for $e$-MERLIN, thus scheduling will not be before 2010. Complimentary A-array EVLA data will be sought at both L and C-Band. In addition, new ultra-deep EVN observations are planned.

Results from $e$-MERGE are likely to be made public in a series of staged releases - the full sensitivity over the complete area will take time to achieve. 


\section{References}

[1] Beswick, R., Muxlow, T. W. B., Thrall, H., Richards, A. M. S. \& Garrington, S. T., An evolution of the infrared-radio correlation at very low flux densities?, 2008 MNRAS, 385, 1143.

[2] Casey, C. M., Impey, C. D.; Trump, J. R., Gabor, J., Abraham, R. G., Capak, P., Scoville, N. Z., Brusa, M., \& Schinnerer, E., Optical Selection of Faint Active Galactic Nuclei in the COSMOS Field, 2008, ApJS, 177, 131.

[3] Chapman, S. C., Smail, I., Windhorst, R., Muxlow, T. W.B., \& Ivison, R. J., Evidence for Extended, Obscured Starbursts in Submillimeter Galaxies, 2004, ApJ, 611, 732.

[4] Garrett, M. A., Muxlow, T. W. B., Garrington, S. T., Alef, W., Alberdi, A., van Langevelde, H. J., Venturi, T., Polatidis, A. G., Kellermann, K. I., Baan, W. A., \& 3 coauthors, AGN and starbursts at high redshift: High resolution EVN radio observations of the Hubble Deep Field, 2001, A\&A, 366, L5.

[5] Haarsma, D.B., Partridge, R. B., Windhorst, R. A., \& Richards, E. A., Faint Radio Sources and Star Formation History, 2000, ApJ, 544,641.

[6] Hopkins, A. M., On the Evolution of Star-forming Galaxies, 2004, ApJ, 615,209.

[7] Lilly, S.J., Le Fevre, O., Hammer, F., Crampton, D., The Canada-France Redshift Survey: The Luminosity Density and Star Formation History of the Universe to Z approximately 1, 1996, ApJ, 460, 1L.

[8] Lilly, S.J., Schade, D., Ellis, R., Le Fevre, O., Brinchmann, J., Tresse, L., Abraham, R., Hammer, F., Crampton, D., Colless, M., \& 3 coauthors, Hubble Space Telescope Imaging of the CFRS and LDSS Redshift Surveys. II. Structural Parameters and the Evolution of Disk Galaxies to Z approximately 1, 1998, ApJ, 500, 75L.

[9] Madau, P., Ferguson, H. C., Dickinson, M. E., Giavalisco, M., S., Charles C., \& Fruchter, A., Highredshift galaxies in the Hubble Deep Field: colour selection and star formation history to z 4, 1996, MNRAS, 283, 1388.

[10] Menanteau, F., Abraham, R. G., \& Ellis, R. S., Evidence for evolving spheroidals in the Hubble Deep Fields North and South, 2001, MNRAS, 322, 1.

[11] Muxlow, T. W. B., Richards, A. M. S., Garrington, S. T., Wilkinson, P. N., Anderson, B., Richards, E. A., Axon, D. J., Fomalont, E. B., Kellermann, K. I., Partridge, R. B., \& Windhorst, R. A., High-resolution studies of radio sources in the Hubble Deep and Flanking Fields, 2005 MNRAS, 358, 1159.

[12] Muxlow, T. W. B., Beswick, R. J., Thrall, H., Richards, A. M. S., Garrington, S. T., \& Pedlar, A., The Statistical Properties of the Very Weak Radio Source Population in the GOODS/ACS HDF-N Region, 2007, ASPC 380, 199.

[13] Seymour, N., Dwelly, T., Moss, D., McHardy, I., Zoghbi, A., Rieke, G., Page, M., Hopkins, A., \& Loaring, The star formation history of the Universe as revealed by deep radio observations, N., 2008, MNRAS, 386, 1695. 